\title{
Reliability Analysis of the Proportional Mean Residual Life Order
}

\author{
M. Kayid, ${ }^{1,2}$ S. Izadkhah, ${ }^{3}$ and H. Alhalees ${ }^{1}$ \\ ${ }^{1}$ Department of Statistics and Operations Research, College of Science, King Saud University, Riyadh 11451, Saudi Arabia \\ ${ }^{2}$ Department of Mathematics, Faculty of Science, Suez University, Suez 41522, Egypt \\ ${ }^{3}$ School of Mathematical Sciences, Ferdowsi University of Mashhad, Mashhad 91779, Iran \\ Correspondence should be addressed to M. Kayid; el_kayid2000@yahoo.com
}

Received 4 April 2014; Accepted 2 August 2014; Published 28 August 2014

Academic Editor: Shaomin Wu

Copyright (C) 2014 M. Kayid et al. This is an open access article distributed under the Creative Commons Attribution License, which permits unrestricted use, distribution, and reproduction in any medium, provided the original work is properly cited.

The concept of mean residual life plays an important role in reliability and life testing. In this paper, we introduce and study a new stochastic order called proportional mean residual life order. Several characterizations and preservation properties of the new order under some reliability operations are discussed. As a consequence, a new class of life distributions is introduced on the basis of the anti-star-shaped property of the mean residual life function. We study some reliability properties and some characterizations of this class and provide some examples of interest in reliability.

\section{Introduction}

Stochastic orders have shown that they are very useful in applied probability, statistics, reliability, operation research, economics, and other related fields. Various types of stochastic orders and associated properties have been developed rapidly over the years. Let $X$ be a nonnegative random variable which denotes the lifetime of a system with distribution function $F$, survival function $\bar{F}=1-F$, and density function $f$. The conditional random variable $X_{t}=(X-t \mid X>t), t \geq$ 0 , is known as the residual life of the system after $t$ given that it has already survived up to $t$. The mean residual life (MRL) function of $X$ is the expectation of $X_{t}$, which is given by

$$
\mu_{X}(t)= \begin{cases}\int_{t}^{\infty} \frac{\bar{F}(u)}{\bar{F}(t)} d u, & t>0, \\ 0, & t \leq 0 .\end{cases}
$$

The MRL function is an important characteristic in various fields such as reliability engineering, survival analysis, and actuarial studies. It has been extensively studied in the literature especially for binary systems, that is, when there are only two possible states for the system as either working or failed. Another useful reliability measure is the hazard rate (HR) function of $X$ which is given by

$$
r_{X}(t)=\frac{f(t)}{\bar{F}(t)}, \quad t \geq 0
$$

The HR function is particularly useful in determining the appropriate failure distributions utilizing qualitative information about the mechanism of failure and for describing the way in which the chance of experiencing the event changes with time. In replacement and repair strategies, although the shape of the HR function plays an important role, the MRL function is found to be more relevant than the HR function because the former summarizes the entire residual life function whereas the latter involves only the risk of instantaneous failure at some time $t$. For an exhaustive monograph on the MRL and HR functions and their reliability analysis, we refer the readers to Ramos-Romero and SordoDíaz [1], Belzunce et al. [2], and Lai and Xie [3]. Based on the MRL function, a well-known MRL order has been introduced and studied in the literature. Gupta and Kirmani [4] and Alzaid [5] were among the first who proposed the MRL order. Over the years, many authors have investigated reliability properties and applications of the MRL order in reliability and survival analysis (cf. Shaked and Shanthikumar [6] and 
Müller and Stoyan [7]). On the other hand, the proportional stochastic orders are considered in the literature to generalize some existing notions of stochastic comparisons of random variables. Proportional stochastic orders as extended versions of the existing common stochastic orders in the literature were studied by some researchers such as Ramos-Romero and Sordo-Díaz [1] and Belzunce et al. [2]. Recently, Nanda et al. [8] gave an effective review of the different partial ordering results related to the MRL order and studied some reliability models in terms of the MRL function.

The purpose of this paper is to propose a new stochastic order called proportional mean residual life (PMRL) order which extends the MRL order to a more general setting. Some implications, characterization properties, and preservation results under weighted distributions of this new order including its relationships with other well-known orders are derived. In addition, two characterizations of this order based on residual life at random time and the excess lifetime in renewal processes are obtained. As a consequence, a new class of lifetime distributions, namely, anti-star-shaped mean residual life (ASMRL) class of life distribution, which is closely related to the concept of the PMRL order, is introduced and studied. A number of useful implications, characterizations, and examples for this class of life distributions are discussed along with some reliability applications. The paper is organized as follows. The precise definitions of some stochastic orders as well as some classes of life distributions which will be used in the sequel are given in Section 2. In that section, the PMRL order is introduced and studied. Several characterizations and preservation properties of this new order under some reliability operations are discussed. In addition, to illustrate the concepts, some applications in the context of reliability theory are included. In Section 3, the ASMRL class of life distributions is introduced and studied. Finally, in Section 4, we give a brief conclusion and some remarks of the current research and its future.

Throughout this paper, the term increasing is used instead of monotone nondecreasing and the term decreasing is used instead of monotone nonincreasing. Let us consider two random variables $X$ and $Y$ having distribution functions $F$ and $G$, respectively, and denote by $\bar{F}(f)$ and $\bar{G}(g)$ their respective survival (density) functions. We also assume that all random variables under consideration are absolutely continuous and have 0 as the common left endpoint of their supports, and all expectations are implicitly assumed to be finite whenever they appear. In addition, we use the notations $\mathbb{R}=(-\infty, \infty), \mathbb{R}^{+}=(0, \infty), \stackrel{s t}{=}$ denotes the equality in distribution, and $X_{v}$ is the weighted version of $X$ according to the weight $v$.

\section{Proportional Mean Residual Life Order}

For ease of reference, before stating our main results, let us recall some stochastic orders, classes of life distributions, and dependence concepts which will be used in the sequel.

Definition 1. The random variable $X$ is said to be smaller than $Y$ in the (i) HR order (denoted as $X \leq_{\mathrm{HR}} Y$ ) if

$$
\frac{\bar{G}(t)}{\bar{F}(t)} \text { is increasing in } t \in \mathbb{R}^{+},
$$

(ii) reversed hazard $(\mathrm{RH})$ order (denoted as $X \leq_{\mathrm{RH}} Y$ ) if

$$
\frac{f(x)}{F(x)} \leq \frac{g(x)}{G(x)}, \quad \forall x \in \mathbb{R}^{+},
$$

which denotes the reversed hazard $(\mathrm{RH})$ rate order,

(iii) MRL order (denoted as $X \leq_{\mathrm{MRL}} Y$ ) if

$$
\frac{\int_{t}^{\infty} \bar{G}(u) d u}{\int_{t}^{\infty} \bar{F}(u) d u} \text { is increasing in } t \in \mathbb{R}^{+} .
$$

Definition 2 (Lai and Xie [3]). The nonnegative random variable $X$ is said to have a decreasing mean residual life (DMRL) whenever the MRL of $X$ is decreasing.

Definition 3 (Lariviere and Porteus [9]). The nonnegative random variable $X$ is said to have an increasing generalized failure rate (IGFR) whenever the generalized failure rate function $\beta_{X}$ of $X$ which is given by $\beta_{X}(x)=x r_{X}(x)$ is increasing in $x \geq 0$.

Note that, in view of a result in Lariviere [10], $X$ has IGFR property if and only if $x X \leq_{\mathrm{HR}} X$, for all $x \in(0,1]$, or equivalently if $X \leq_{\mathrm{HR}} x X$, for any $x \geq 1$.

Definition 4 (Karlin [11]). A nonnegative measurable function $h(x, y)$ is said to be totally positive of order $2\left(\mathrm{TP}_{2}\right)$ in $x \in \mathbb{R}$ and $y \in \mathbb{R}$, whenever

$$
\left|\begin{array}{cc}
h\left(x_{1}, y_{1}\right) & h\left(x_{1}, y_{2}\right) \\
h\left(x_{2}, y_{1}\right) & \left(x_{2}, y_{2}\right)
\end{array}\right| \geq 0, \quad \forall x_{1} \leq x_{2}, \quad y_{1} \leq y_{2}
$$

Definition 5 (Shaked and Shanthikumar [6]). A nonnegative function $\psi$ is said to be anti-star-shaped on a set $A \subseteq \mathbb{R}^{+}$ if $\psi(\alpha x) \geq \alpha \psi(x)$, for all $x$ on $A$ and for every $\alpha \epsilon$ $[0,1]$. Equivalently, $\psi$ is anti-star-shaped on $A$ if $\psi(x) / x$ is nonincreasing in $x \in A$.

Below, we present the definition of the proportional hazard rate (PHR) order and its related proportional aging class.

Definition 6 (see Belzunce et al. [2]). Let $X$ and $Y$ be two nonnegative continuous random variables. It is said that

(i) $X$ is smaller than $Y$ in the PHR order (denoted as $\left.X \leq_{\mathrm{P}-\mathrm{HR}} Y\right)$ if $x X \leq_{\mathrm{HR}} Y$, for all $x \in(0,1]$,

(ii) $X$ is increasing proportional hazard rate (IPHR), if $x X \leq_{\mathrm{HR}} X$, for all $x \in(0,1]$.

Consider the situation wherein $X$ denotes the risk that the direct insurer faces and $\phi$ the corresponding reinsurance contract. One important reinsurance agreement is quotashare treaty defined as $\phi(X)=x X$, for $x \in(0,1]$. The random variable $Y$ denotes the risk that an independent insurer 
faces. Insurers sometimes seek a quota-share treaty when they require financial support from their reinsurers, thus maintaining an adequate relation between net income and capital reserves. Motivated by this, we propose the following new stochastic order.

Definition 7. Let $X$ and $Y$ be two nonnegative random variables. The random variable $X$ is smaller than $Y$ in the PMRL order (denoted as $X \leq_{\mathrm{P}-\mathrm{MRL}} Y$ ), if $x X \leq_{\mathrm{MRL}} Y$, for all $x \in(0,1]$.

Remark 8. Note that $X \leq_{\mathrm{P}-\mathrm{MRL}} Y \Leftrightarrow x X \leq_{\mathrm{MRL}} Y$, for all $x \in$ $(0,1]$, thus; with $x=1$ we have $X \leq_{\mathrm{MRL}} Y$, that is; the PMRL order is stronger than the MRL order.

The first results of this section provide an equivalent condition for the PMRL order.

Theorem 9. The following assertions are equivalent:

(i) $X \leq_{P-M R L} Y$;

(ii) $x \mu_{X}(t) \leq \mu_{Y}(x t)$, for all $t \geq 0$, and each $x \in(0,1]$;

(iii) $\int_{x t}^{\infty} \bar{G}(u) d u / \int_{t}^{\infty} \bar{F}(u) d u$ is increasing in $t$, for all $x \in$ $(0,1]$.

Proof. First, we prove that (i) and (ii) are equivalent. Note that the MRL of $x X$ as a function of $s$ is given by $x \mu_{X}(s / x)$, for all $s \geq 0$ and for any $x \in(0,1]$. Now, we have $X \leq_{\mathrm{P}-\mathrm{MRL}} Y$ if, for all $x \in(0,1]$, it holds that

$$
\begin{aligned}
& x X \leq_{\mathrm{MRL}} Y \\
& \Longleftrightarrow x \mu_{X}\left(\frac{s}{x}\right) \leq \mu_{Y}(s), \quad \forall s \geq 0, \\
& \Longleftrightarrow x \mu_{X}(t) \leq \mu_{Y}(x t), \quad \forall t \geq 0, \\
& \text { where } t=\frac{s}{x} .
\end{aligned}
$$

To prove that (ii) and (iii) are equivalent, we have

$$
\begin{aligned}
& \frac{d}{d t} \frac{\int_{x t}^{\infty} \bar{G}(u) d u}{\int_{t}^{\infty} \bar{F}(u) d u} \\
& =\frac{\bar{F}(t) \int_{x t}^{\infty} \bar{G}(u) d u-x \bar{G}(x t) \int_{t}^{\infty} \bar{F}(u) d u}{\left[\int_{t}^{\infty} \bar{F}(u) d u\right]^{2}} \\
& =\frac{\bar{F}(t) \bar{G}(x t)}{\left[\int_{t}^{\infty} \bar{F}(u) d u\right]^{2}} \times\left[\mu_{Y}(x t)-x \mu_{X}(t)\right] .
\end{aligned}
$$

It is obvious that the last term is nonnegative if and only if $x \mu_{X}(t) \leq \mu_{Y}(x t)$, for all $t \geq 0$ and for any $x \in(0,1]$.

In the context of reliability engineering and survival analysis, weighted distributions are of tremendous practical importance (cf. Jain et al. [12], Bartoszewicz and Skolimowska [13], Misra et al. [14], Izadkhah and Kayid [15], and Kayid et al. [16]). In renewal theory the residual lifetime has a limiting distribution that is a weighted distribution with the weight function equal to the reciprocal of the HR function. Some of the well-known and important distributions in statistics and applied probability may be expressed as weighted distributions such as truncated distributions, the equilibrium renewal distribution, distributions of order statistics, and distributions arisen in proportional hazards and proportional reversed hazards models. Recently, Izadkhah et al. [17] have considered the preservation property of the MRL order under weighted distributions. Here we develop a similar preservation property for the PMRL order under weighted distributions. For two weight functions $w_{1}$ and $w_{2}$, assume that $X_{w_{1}}$ and $Y_{w_{2}}$ denote the weighted versions of the random variables of $X$ and $Y$, respectively, with respective density functions

$$
f_{1}(x)=\frac{w_{1}(x) f(x)}{v_{1}}, \quad g_{1}(x)=\frac{w_{2}(x) g(x)}{v_{2}},
$$

where $0<v_{1}=E\left(w_{1}(X)\right)<\infty$ and $0<v_{2}=E\left(w_{2}(Y)\right)<\infty$. Let $B_{1}(x)=E\left(w_{1}(X) \mid X>x\right)$ and $B_{2}(x)=E\left(w_{2}(Y) \mid Y>\right.$ $x)$. Then survival functions of $X_{w_{1}}$ and $Y_{w_{2}}$ are, respectively, given by

$$
\bar{F}_{1}(x)=\frac{B_{1}(x) \bar{F}(x)}{\nu_{1}}, \quad \bar{G}_{1}(x)=\frac{B_{2}(x) \bar{G}(x)}{\nu_{2}} .
$$

First, we consider the following useful lemma which is straightforward and hence the proof is omitted.

Lemma 10. Let $X$ be a nonnegative absolutely continuous random variable. Then, for any weight function $w_{1}$,

$$
(x X)_{v} \stackrel{s t}{=} x X_{w_{1}}, \quad \forall x>0,
$$

where $v$ is a weight function of the form $v(t)=w_{1}(t / x)$.

Theorem 11. Let $B_{2}$ be an increasing function and let $B_{2}(s x) / B_{1}(s)$ increase in $s \geq 0$, for all $x \in(0,1]$. Then

$$
X \leq_{P-M R L} Y \Longrightarrow X_{w_{1}} \leq_{P-M R L} Y_{w_{2}} .
$$

Proof. Let $x \in(0,1]$ be fixed. Then, $X \leq_{\mathrm{P}-\mathrm{MRL}} Y$ gives $x X \leq_{\mathrm{MRL}} Y$. We know by assumption that $B_{2}$ is increasing and the ratio

$$
\begin{aligned}
& \frac{E\left(w_{2}(Y) \mid Y>t\right)}{E(v(x X) \mid x X>t)} \\
& \quad=\frac{E\left(w_{2}(Y) \mid Y>t\right)}{E\left(w_{1}(X) \mid X>t / x\right)} \\
& \quad=\frac{E\left(w_{2}(Y) \mid Y>s x\right)}{E\left(w_{1}(X) \mid X>s\right)}=\frac{B_{2}(s x)}{B_{1}(s)}
\end{aligned}
$$

is increasing in $t \geq 0$, when $s=t / x$. In view of Theorem 2 in Izadkhah et al. [17], we conclude that $(x X)_{v} \leq_{\mathrm{MRL}} Y_{w_{2}}$. Because of Lemma 10 and because the equality in distribution of $(x X)_{v}$ and $x X_{w_{1}}$ implies the equality in their MRL functions, it follows that $x X_{w_{1}} \leq_{\mathrm{MRL}} Y_{w_{2}}$. So, for all $x \in(0,1]$ we have $x X_{w_{1}} \leq_{\mathrm{MRL}} Y_{w_{2}}$ which means that $X_{w_{1}} \leq_{\mathrm{P}-\mathrm{MRL}} Y_{w_{2}}$. 
On the other hand, in many reliability engineering problems, it is interesting to study $X_{Y}=[X-Y \mid X>Y]$, the residual life of $X$ with a random age $Y$. The residual life at random time (RLRT) represents the actual working time of the standby unit if $X$ is regarded as the total random life of a warm standby unit with its age $Y$. For more details about RLRT we refer the readers to Yue and Cao [18], Li and Zuo [19], and Misra et al. [20], among others. Suppose that $X$ and $Y$ are independent. Then, the survival function of $X_{Y}$, for any $x \geq 0$, is given by

$$
P\left(X_{Y}>x\right)=\frac{\int_{0}^{\infty} \bar{F}(x+y) d G(y)}{\int_{0}^{\infty} \bar{F}(y) d G(y)} .
$$

Theorem 12. Let $X$ and $Y$ be two nonnegative random variables. $X_{Y} \leq_{P-M R L} X$ for any $Y$ which is independent of $X$, if and only if

$$
X_{t} \leq_{P-M R L} X, \quad \forall t \geq 0 .
$$

Proof. To prove the "if" part, let $X_{t} \leq_{\mathrm{P}-\mathrm{MRL}} X$ for all $t \geq 0$. It then follows that, for all $s>0$ and $x \in(0,1]$,

$$
\int_{s}^{\infty} x \bar{F}(t+u) d u \leq \frac{\bar{F}(t+s)}{\bar{F}(s x)} \int_{s x}^{\infty} \bar{F}(u) d u .
$$

By integrating both sides of (16) with respect to $t$ through the measure $G$, we have

$$
\begin{array}{r}
\int_{0}^{\infty} \int_{s}^{\infty} x \bar{F}(t+u) d u d G(t) \\
\leq \int_{0}^{\infty}\left[\frac{\bar{F}(t+s)}{\bar{F}(s x)} \int_{s x}^{\infty} \bar{F}(u) d u\right] d G(t) \\
=\int_{0}^{\infty} \bar{F}(t+s) d G(t) \frac{\int_{s x}^{\infty} \bar{F}(u) d u}{\bar{F}(s x)}, \\
\forall s \geq 0, \quad x \in(0,1],
\end{array}
$$

which is equivalent to saying that $X_{Y} \leq_{\mathrm{P}-\mathrm{MRL}} X$, for all $Y$ 's that are independent of $X$. For the "only if" part, suppose that $X_{Y} \leq_{\mathrm{P}-\mathrm{MRL}} X$ holds for any nonnegative random variable $Y$. Then $X_{t} \leq_{\mathrm{P}-\mathrm{MRL}} X$, for all $t \geq 0$, follows by taking $Y$ as a degenerate random variable.

Let $\left\{X_{n}, n=1,2, \ldots\right\}$ be a sequence of mutually independent and identically distributed (i.i.d.) nonnegative random variables with common distribution function $F$. For $n \geq 1$, denote $S_{n}=\sum_{i=1}^{n} X_{i}$ which is the time of the $n$th arrival and $S_{0}=0$, and let $N(t)=\operatorname{Sup}\left\{n: S_{n} \leq t\right\}$ represent the number of arrivals during the interval $[0, t]$. Then, $N=$ $\{N(t), t \geq 0\}$ is a renewal process with underlying distribution $F$ (see Ross [21]). Let $\gamma(t)$ be the excess lifetime at time $t \geq 0$; that is, $\gamma(t)=S_{N(t)+1}-t$. In this context we denote the renewal function by $M(t)=E[N(t)]$ which satisfies the following well-known fundamental renewal equation:

$$
M(t)=F(t)+\int_{0}^{t} F(t-y) d M(y), \quad t \geq 0 .
$$

According to Barlow and Proschan [22], it holds for all $t \geq 0$ and $x \geq 0$ that

$$
P(\gamma(t)>x)=\bar{F}(t+x)+\int_{0}^{t} \bar{F}(t+x-z) d M(z) .
$$

In the literature, several results have been given to characterize the stochastic orders by the excess lifetime in a renewal process. Next, we investigate the behavior of the excess lifetime of a renewal process with respect to the PMRL order.

Theorem 13. If $X_{t} \leq_{P-M R L} X$, for all $t \geq 0$, then $\gamma(t) \leq_{P-M R L} \gamma(0)$ for all $t \geq 0$.

Proof. First note that $X_{t} \leq_{\mathrm{P}-\mathrm{MRL}} X$, for all $t \geq 0$, if and only if for any $t \geq 0, s>0$, and $x \in(0,1]$

$$
\int_{s}^{\infty} x \bar{F}(t+u) d u \leq \bar{F}(t+s) \frac{\int_{s x}^{\infty} \bar{F}(u) d u}{\bar{F}(s x)} .
$$

In view of the identity of (19) and the inequality in (20) we can get

$$
\begin{aligned}
& \int_{s}^{\infty} x P(\gamma(t)>u) d u \\
& =\int_{s}^{\infty} x \bar{F}(t+u) d u \\
& +\int_{0}^{t} \int_{s}^{\infty} x \bar{F}(t-y+u) d u d M(y) \\
& \leq \int_{s}^{\infty} x \bar{F}(t+u) d u \\
& +\int_{0}^{t}\left[\frac{\bar{F}(t-y+s)}{\bar{F}(s x)} \int_{s x}^{\infty} \bar{F}(u) d u\right] d M(y) \\
& =\int_{s}^{\infty} x \bar{F}(t+u) d u \\
& +\frac{\int_{s x}^{\infty} \bar{F}(u) d u}{\bar{F}(s x)}[P(\gamma(t)>s)-\bar{F}(t+s)] \\
& \leq \frac{\int_{s x}^{\infty} \bar{F}(u) d x}{\bar{F}(s x)}[\bar{F}(t+s)] \\
& +\frac{\int_{s x}^{\infty} \bar{F}(u) d u}{\bar{F}(s x)}[P(\gamma(t)>s)-\bar{F}(t+s)] \\
& =\frac{\int_{s x}^{\infty} \bar{F}(u) d u}{\bar{F}(s x)} P(\gamma(t)>s) .
\end{aligned}
$$

Hence, it holds that, for all $t \geq 0, s>0$ and for any $x \in(0,1]$,

$$
\frac{\int_{s}^{\infty} x P(\gamma(t)>u) d u}{P(\gamma(t)>s)} \leq \frac{\int_{s x}^{\infty} \bar{F}(u) d u}{\bar{F}(s x)},
$$

which means $\gamma(t) \leq_{\mathrm{P}-\mathrm{MRL}} \gamma(0)$ for all $t \geq 0$. 


\section{Anti-Star-Shaped Mean Residual Life Class}

Statisticians and reliability analysts have shown a growing interest in modeling survival data using classifications of life distributions by means of various stochastic orders. These categories are useful for modeling situations, maintenance, inventory theory, and biometry. In this section, we propose a new class of life distributions which is related to the MRL function. We study some characterizations, preservations, and applications of this new class. Some examples of interest in the context of reliability engineering and survival analysis are also presented.

Definition 14. The lifetime variable $X$ is said to have an antistar-shaped mean residual life (ASMRL), if the MRL function of $X$ is anti-star-shaped.

It is simply derived that $X \in$ ASMRL whenever $\mu_{X}(t) / t$ is decreasing in $t>0$. Useful description and motivation for the definition of the ASMRL class which is due to Nanda et al. [8] are the following. Consider a situation in which $X$ represents the risk that the direct insurer faces and $\phi$ the corresponding reinsurance contract. The ASMRL class provides that the quota-share treaty related to a risk is less than risk itself in the sense of the MRL order. In what follows, we focus on the ASMRL class as a weaker class than the DMRL class to get some basic results. First, consider the following characterization property which can be immediately obtained by Theorem 9(ii).

Theorem 15. The lifetime random variable $X$ is ASMRL if and only if $X \leq_{P-M R L} X$.

Theorem 16. The lifetime random variable $X$ is ASMRL if and only if

$$
\theta_{1} X \leq_{M R L} \theta_{2} X, \quad \text { for any } \theta_{1} \leq \theta_{2} \in \mathbb{R}^{+} .
$$

Proof. Denote $X\left(\theta_{i}\right)=\theta_{i} X$, for $i=1,2$. The MRL function of $X\left(\theta_{i}\right)$ is then given by $\mu_{X\left(\theta_{i}\right)}(t)=\theta_{i} \mu_{X}\left(t / \theta_{i}\right)$, for all $t \geq 0$ and $i=1$, 2.In view of the fact that $\theta_{1} X \leq_{\mathrm{MRL}} \theta_{2} X$, for all $\theta_{1} \leq \theta_{2} \in$ $\mathbb{R}^{+}$, if and only if

$$
\theta_{1} \mu_{X}\left(\frac{t}{\theta_{1}}\right) \leq \theta_{2} \mu_{X}\left(\frac{t}{\theta_{2}}\right), \quad \text { for any } \theta_{1} \leq \theta_{2} \in \mathbb{R}^{+} .
$$

By taking $x=\theta_{1} / \theta_{2}$ and $s=t / \theta_{1}$ the above inequality is equivalent to saying that $x \mu_{X}(s) \leq \mu_{X}(x s)$, for all $s \geq 0$ and for any $x \in(0,1]$. This means that $X$ is ASMRL.

Remark 17. The result of Theorem 16 indicates that the family of distributions $F_{\theta}(x)=F(x / \theta), \theta>0$, is stochastically increasing in $\theta$ with respect to the MRL order if and only if the distribution $F$ has an anti-star-shaped MRL function. Another conclusion of Theorem 16 is to say that $X \leq_{\mathrm{P}-\mathrm{MRL}} X$ if and only if $X \leq_{\mathrm{MRL}} x X$, for all $x \in[1, \infty)$.

Theorem 18. If $X \leq_{M R L} Y$ and if either $X$ or $Y$ has an anti-starshaped MRL function, then $X \leq_{P-M R L} Y$.

Proof. Let $X \leq_{\mathrm{MRL}} Y$ and let $X$ be ASMRL. Then, we have

$$
x X \leq_{\mathrm{MRL}} X \leq_{\mathrm{MRL}} Y, \quad \forall x \in(0,1] .
$$

Hence it holds that $x X \leq_{\mathrm{MRL}} Y$, for all $x \in(0,1]$, which means $X \leq_{\mathrm{P}-\mathrm{MRL}} Y$. The proof of the result when $Y$ is ASMRL is similar by taking the fact that $Y$ is ASMRL if and only if $Y \leq_{\text {MRL }} \alpha Y$, for all $\alpha \geq 1$, into account. Note also that $X \leq_{\mathrm{P}-\mathrm{MRL}} Y$ if and only if $X \leq_{\mathrm{MRL}} \alpha Y$, for any $\alpha \geq 1$.

The following counterexample shows that the MRL order does not generally imply the ASMRL order and hence the sufficient condition in Theorem 18 cannot be removed.

Counter Example 1. Let $X$ have MRL $\mu_{X}(t)=(t-1 / 2)^{2}$, for $t \in[0, \infty)$, and let $Y$ have MRL $\mu_{Y}(t)=1 / 6$, for $t \in[0,1 / 6]$, and $\mu_{Y}(t)=3(t-1 / 2)^{2} / 2$, for $t \in(1 / 6, \infty)$. These MRL functions are readily shown not to be ASMRL. We can also see that $\mu_{X}(t) \leq \mu_{Y}(t)$, for all $t \geq 0$; that is, $X \leq_{\mathrm{MRL}} Y$. It can be easily checked now that $x X \Varangle_{\mathrm{MRL}} Y$, for $x=1 / 4$, which means that $X \Varangle_{\text {P-MRL }} Y$.

As an obvious conclusion of Theorem 18 above and Theorem 2.9 in Nanda et al. [8], if $X$ is DMRL, then $X$ is ASMRL. The next result presents another characterization of the ASMRL class.

Theorem 19. A lifetime random variable $X$ is ASMRL if and only if $Z X \leq_{M R L} X$, for each random variable $Z$ with $S_{Z}=(0,1]$, which is independent of $X$.

Proof. To prove the "if" part, note that $\mu_{z X}(t)=z \mu_{X}(t / z)$, for each $z \in(0,1]$ and any $t>0$. Take $Z=z$, for each $z \in$ $(0,1]$ one at a time, as a degenerate random variable implying $z X \leq_{\mathrm{MRL}} X$, for all $z \in(0,1]$, which means $X \in$ ASMRL. For the "only if" part, assume that $Z$ has distribution function $G$. From the assumption and the well-known Fubini theorem, for all $x>0$, it follows that

$$
\begin{aligned}
\mu_{Z X}(x) & =\frac{\int_{x}^{\infty} P(Z X>u) d u}{P(Z X>x)} \\
& =\frac{\int_{x}^{\infty} \int_{0}^{1} \bar{F}(u / z) d G(z) d u}{\int_{0}^{1} \bar{F}(x / z) d G(z)} \\
& =\frac{\int_{0}^{1} \int_{x}^{\infty} \bar{F}(u / z) d u d G(z)}{\int_{0}^{1} \bar{F}(x / z) d G(z)} \\
& =\frac{\int_{0}^{1} z m_{X}(x / z) \bar{F}(x / z) d G(z)}{\int_{0}^{1} \bar{F}(x / z) d G(z)} \\
& \leq \frac{\int_{0}^{1} m_{X}(x) \bar{F}(x / z) d G(z)}{\int_{0}^{1} \bar{F}(x / z) d G(z)} \\
& =\mu_{X}(x) .
\end{aligned}
$$

That is, $Z X \leq_{\mathrm{MRL}} X$.

The following result presents a sufficient condition for a probability distribution to be ASMRL. 
Theorem 20. Let the lifetime random variable $X$ be IGFR. Then, $X$ is ASMRL.

Proof. Recall that $X$ is IGFR if and only if $x r_{X}(x)$ is increasing in $x \geq 0$. Because of the identity

$$
t \bar{F}(t)=\int_{t}^{\infty} u f(u) d u-\int_{t}^{\infty} \bar{F}(u) d u \quad \forall t>0,
$$

we can write, for all $t>0$,

$$
\begin{aligned}
\frac{\mu_{X}(t)}{t} & =\frac{\int_{t}^{\infty} \bar{F}(u) d u}{t \bar{F}(t)} \\
& =\frac{\int_{t}^{\infty} \bar{F}(u) d u}{\int_{t}^{\infty} u f(u) d u-\int_{t}^{\infty} \bar{F}(u) d u} \\
& =\frac{1}{\left(\int_{t}^{\infty} u f(u) d u / \int_{t}^{\infty} \bar{F}(u) d u\right)-1} .
\end{aligned}
$$

Thus, $\mu_{X}(t) / t$ is decreasing in $t>0$ if and only if the ratio

$$
\frac{\int_{t}^{\infty} u f(u) d u}{\int_{t}^{\infty} \bar{F}(u) d u} \text { is increasing in } t>0 .
$$

Let

$$
\rho(i, t)=\int_{0}^{\infty} \phi(i, u) \psi(u, t) d u
$$

as a function of $i=1,2$ and of $t>0$, where

$$
\begin{gathered}
\phi(i, u)= \begin{cases}u f(u), & \text { if } i=2, \\
\bar{F}(u), & \text { if } i=1,\end{cases} \\
\psi(u, t)= \begin{cases}1, & \text { if } u>t, \\
0, & \text { if } u \leq t .\end{cases}
\end{gathered}
$$

Note that the ratio given in (29) is increasing in $t>0$ if and only if $\rho$ is $\mathrm{TP}_{2}$ in $(i, t) \in\{1,2\} \times(0, \infty)$. From the assumption, since $u r_{X}(u)$ is increasing, then $\phi$ is $\mathrm{TP}_{2}$ in $(i, u) \in\{1,2\} \times(0, \infty)$. Also it is easy to see that $\psi$ is $\mathrm{TP}_{2}$ in $(u, t) \in(0, \infty) \times(0, \infty)$. By applying the general composition theorem of Karlin [11] to the equality of (30), the proof is complete.

To demonstrate the usefulness of the ASMRL class in reliability engineering problems, we consider the following examples.

Example 21. The Weibull distribution is one of the most widely used lifetime distributions in reliability engineering. It is a versatile distribution that can take on the characteristics of other types of distributions, based on the value of the shape parameter. Let $X$ have the Weibull distribution with survival function

$$
\bar{F}(x)=\exp \left(-(\lambda x)^{\alpha}\right), \quad x \geq 0, \alpha>0, \lambda>0 .
$$

The HR function is given by $r_{X}(x)=\alpha \lambda^{\alpha} x^{\alpha-1}$. Thus we have $x r_{X}(x)=\alpha(\lambda x)^{\alpha}$ which is increasing in $x>0$ for all parameter values and hence according to Theorem $20 X$ is ASMRL.
Example 22. The generalized Pareto distribution has been extensively used in reliability studies when robustness is required against heavier tailed or lighter tailed alternatives to an exponential distribution. Let $X$ have generalized Pareto distribution with survival function

$$
\bar{F}(x)=\left(\frac{b}{a x+b}\right)^{(1 / a)+1}, \quad x \geq 0, a>0, b>0
$$

The HR function is given by $r_{X}(x)=(1+a) /(a x+b)$. Thus we get $x r_{X}(x)=(1+a) x /(a x+b)$ which is increasing in $x$ for all parameter values and so Theorem 20 concludes that $X$ is ASMRL.

Example 23. Let $T_{i}$ be a lifetime variable having survival function given by $\bar{F}_{i}(t)=E\left(\bar{G}\left(Z_{i} t\right)\right), t \geq 0$, where $Z_{i}$ is a nonnegative random variable and $\bar{G}$ is the survival function of a lifetime variable $Y$, for each $i=1,2$. This is called scale change random effects model in Ling et al. [23]. Noting the fact that $Y \leq_{\mathrm{MRL}} k Y$, for all $k \geq 1$, is equivalent to saying that $Y$ is ASMRL, according to Theorem 3.10 of Ling et al. [23] if $Z_{1} \leq_{\mathrm{RH}} Z_{2}$ and $Y$ is ASMRL, then $T_{1} \geq_{\mathrm{MRL}} T_{2}$.

In the context of reliability theory, shock models are of great interest. The system is assumed to have an ability to withstand a random number of these shocks, and it is commonly assumed that the number of shocks and the interarrival times of shocks are $s$-independent. Let $N$ denote the number of shocks survived by the system, and let $X_{j}$ denote the random interarrival time between the $(j-1)$ th and $j$ th shocks. Then the lifetime $T$ of the system is given by $T=\sum_{j=1}^{N} X_{j}$. Therefore, shock models are particular cases of random sums. In particular, if the interarrivals are assumed to be $s$-independent and exponentially distributed (with common parameter $\lambda$ ), then the distribution function of $T$ can be written as

$$
H(t)=\sum_{k=0}^{\infty} \frac{e^{-\lambda t}(\lambda t)^{k}}{k !} P_{k}, \quad t \geq 0
$$

where $P_{k}=P[N \leq k]$ for all $k \in N$ (and $\bar{P}_{0}=1$ ). Shock models of this kind, called Poisson shock models, have been studied extensively. For more details, we refer to Fagiuoli and Pellerey [24], Shaked and Wong [25], Belzunce et al. [26], and Kayid and Izadkhah [27].

In the following, we make conditions on the random number of shocks under which $T$ has ASMRL property. First, let us define the discrete version of the ASMRL class.

Definition 24. A discrete distribution $P_{k}$ is said to have discrete anti-star-shaped mean residual life (D-ASMRL) property if $\sum_{j=k}^{\infty} \bar{P}_{j} / k \bar{P}_{k-1}$ is nonincreasing in $k \in N$.

Theorem 25. If $P_{k}, k \in N$, in (34) is D-ASMRL, then $T$ with the sf $\bar{H}(t)$ as given in (34) is ASMRL. 
Proof. We may note that, for all $t>0$,

$$
\begin{gathered}
t \bar{H}(t)=\frac{1}{\lambda} \sum_{k=0}^{\infty} e^{-\lambda t} \frac{(\lambda t)^{k}}{k !} k \bar{P}_{k-1}, \\
\int_{t}^{\infty} \bar{H}(u) d u=\frac{1}{\lambda} \sum_{k=0}^{\infty} e^{-\lambda t} \frac{(\lambda t)^{k}}{k !} \sum_{j=k}^{\infty} \bar{P}_{j} .
\end{gathered}
$$

Hence $T$ is ASMRL if and only if

$$
\frac{t \bar{H}(t)}{\int_{t}^{\infty} \bar{H}(u) d u}=\frac{\sum_{k=0}^{\infty} e^{-\lambda t}\left((\lambda t)^{k} / k !\right) k \bar{P}_{k-1}}{\sum_{k=0}^{\infty} e^{-\lambda t}\left((\lambda t)^{k} / k !\right) \sum_{j=k}^{\infty} \bar{P}_{j}}
$$

is increasing in $t$, or equivalently if

$$
\Psi(i, t)=\sum_{k=0}^{\infty} \Phi(i, k) \frac{(\lambda t)^{k}}{k !} e^{-\lambda t} \text { is } \mathrm{TP}_{2} \text { in }(i, t),
$$

for $i \in\{1,2\}$ and $t \in \mathbb{R}^{+}$, where

$$
\Phi(i, k)= \begin{cases}\sum_{j=k}^{\infty} \bar{P}_{j}, & \text { if } i=1, \\ k \bar{P}_{k-1}, & \text { if } i=2 .\end{cases}
$$

By the assumption, $\Phi(i, k)$ is $\mathrm{TP}_{2}$ in $(i, k)$, for $i \in\{1,2\}$ and $k \in N$. It is also evident that $e^{-\lambda t}(\lambda t)^{k} / k$ ! is $\mathrm{TP}_{2}$ in $(k, t)$, for $k \in N$ and $t \in \mathbb{R}^{+}$. The result now follows from the general composition theorem of Karlin [11].

Lemma 26. Let $X_{1}, X_{2}, \ldots, X_{n}$ be an i.i.d. sample from $F$ and let $Y_{1}, Y_{2}, \ldots, Y_{n}$ be an i.i.d. sample from $G$. Then $\min \left\{X_{1}, X_{2}, \ldots, X_{n}\right\} \leq_{M R L} \min \left\{Y_{1}, Y_{2}, \ldots, Y_{n}\right\} \quad$ implies $X_{i} \leq_{M R L} Y_{i}, i=1,2, \ldots, n$.

Example 27. Reliability engineers often need to work with systems having elements connected in series. Let $X_{1}, X_{2}, \ldots, X_{n}$ be i.i.d. random lifetimes such that $T=\min \left\{X_{1}, X_{2}, \ldots, X_{n}\right\}$ has the ASMRL property. Then, according to Theorem $15, k T \leq_{\mathrm{MRL}} T$, for all $k \in(0,1]$. This means that

$$
\begin{aligned}
& \min \left\{k X_{1}, k X_{2}, \ldots, k X_{n}\right\} \\
& \quad \leq_{\text {MRL }} \min \left\{X_{1}, X_{2}, \ldots, X_{n}\right\}, \quad \forall k \in(0,1] .
\end{aligned}
$$

By appealing to Lemma 26, it follows that $k X_{i} \leq_{\mathrm{MRL}} X_{i}, i=$ $1,2, \ldots, n$, for all $k \in(0,1]$. That is, $X_{i}, i=1,2, \ldots, n$, is ASMRL. Hence, the ASMRL property passes from the lifetime of the series system to the lifetime of its i.i.d. components.

Accelerated life models relate the lifetime distribution to the explanatory variables (stress, covariates, and regressor). This distribution can be defined by the survival, cumulative distribution, or probability density functions. Nevertheless, the sense of accelerated life models is best seen if they are formulated in terms of the hazard rate function. In the following example, we state an application of Theorem 16 in accelerated life models.
Example 28. Consider $n$ units (not necessarily independent) with lifetimes $T_{i}, i=1,2, \ldots, n$. Suppose that the units are working in a common operating environment, which is represented by a random vector $\Theta=\left(\Theta_{1}, \Theta_{2}, \ldots, \Theta_{n}\right)$, independent of $T_{1}, T_{2}, \ldots, T_{n}$, and has an effect on the units of the form

$$
X_{i}=\frac{T_{i}}{\Theta_{i}}, \quad i=1,2, \ldots, n .
$$

If $\Theta$ has support on $(1, \infty)^{n}$, then the components are working in a harsh environment, and if they have support on $(0,1)^{n}$, then the components are working in a gentler environment (see Ma [28]). In a harsh environment let $T_{j} \in$ ASMRL for some $j=1,2, \ldots, n$. Then, Theorem 19 states that, for each $Z$ with support on $[0,1]$, we must have $Z T_{j} \leq_{\mathrm{MRL}} T_{j}$. Thus, by taking $Z=1 / \Theta_{j}$, we must have $T_{j} / \Theta_{j} \leq_{\mathrm{MRL}} T_{j}$. Hence, by (40) it stands that $X_{j} \leq_{\mathrm{MRL}} T_{j}$. With a similar discussion, in a gentler environment if $X_{j} \in$ ASMRL for some $j=1,2, \ldots, n$, then we must have $T_{j} \leq_{\mathrm{MRL}} X_{j}$.

In the following we state the preservation property of the ASMRL class under weighted distribution. Let $X$ have density function $f$ and survival function $\bar{F}$. The following result states the preservation of the ASMRL class under weighted distributions. The proof is quite similar to that of Theorem 11 and hence omitted.

Theorem 29. Let $B$ be an increasing function and let $B(s x) / B(s)$ increase in $s \geq 0$, for all $x \in(0,1]$. Then $X$ is $A S M R L$ implying that $X_{w}$ is ASMRL.

\section{Conclusion}

Due to economic consequences and safety issues, it is necessary for the industry to perform systematic studies using reliability concepts. There exist plenty of scenarios where a statistical comparison of reliability measures is required in both reliability engineering and biomedical fields. In this paper, we have proposed a new stochastic order based on the MRL function called proportional mean residual life (PMRL) order. The relationships of this new stochastic order with other well-known stochastic orders are discussed. It was shown that the PMRL order enjoys several reliability properties which provide several applications in reliability and survival analysis. We discussed several characterization and preservation properties of this new order under some reliability operations. To enhance the study, we proposed a new class of life distributions called anti-star-shaped mean residual life (ASMRL) class. Several reliability properties of the new class as well as a number of applications in the context of reliability and survival analysis are included. Our results provide new concepts and applications in reliability, statistics, and risk theory. Further properties and applications of the new stochastic order and the new proposed class can be considered in the future of this research. In particular, the following topics are interesting and still remain as open problems:

(i) closure properties of the PMRL order and the ASMRL class under convolution and coherent structures, 
(ii) discrete version of the PMRL order and enhancing the obtained results related to the D-ASMRL class,

(iii) testing exponentiality against the ASMRL class.

\section{Conflict of Interests}

The authors declare that there is no conflict of interests regarding the publication of this paper.

\section{Acknowledgments}

The authors would like to thank two reviewers for their valuable comments and suggestions, which were helpful in improving the paper. The authors would also like to extend their sincere appreciation to the Deanship of Scientific Research at King Saud University for funding this Research Group (no. RG-1435-036).

\section{References}

[1] H. M. Ramos-Romero and M. A. Sordo-Díaz, "The proportional likelihood ratio order and applications," Questiio, vol. 25, no. 2, pp. 211-223, 2001.

[2] F. Belzunce, J. M. Ruiz, and M. C. Ruiz, "On preservation of some shifted and proportional orders by systems," Statistics and Probability Letters, vol. 60, no. 2, pp. 141-154, 2002.

[3] C. D. Lai and M. Xie, Stochastic Ageing and Dependence for Reliability, Springer, New York, NY, USA, 2006.

[4] R. C. Gupta and S. N. U. Kirmani, "On order relations between reliability measures," Communications in Statistics. Stochastic Models, vol. 3, no. 1, pp. 149-156, 1987.

[5] A. A. Alzaid, "Mean residual life ordering," Statistical Papers, vol. 29, no. 1, pp. 35-43, 1988.

[6] M. Shaked and J. G. Shanthikumar, Stochastic Orders, Springer Series in Statistics, Springer, New York, NY, USA, 2007.

[7] A. Müller and D. Stoyan, Comparison Methods for Stochastic Models and Risks, John Wiley, New York, NY, USA, 2002.

[8] A. K. Nanda, S. Bhattacharjee, and N. Balakrishnan, "Mean residual life function, associated orderings and properties," IEEE Transactions on Reliability, vol. 59, no. 1, pp. 55-65, 2010.

[9] M. A. Lariviere and E. L. Porteus, "Selling to a news vendor: an analysis of price-only contracts," Manufacturing and Service Operations Management, vol. 3, no. 4, pp. 293-305, 2001.

[10] M. A. Lariviere, "A note on probability distributions with increasing generalized failure rates," Operations Research, vol. 54, no. 3, pp. 602-604, 2006.

[11] S. Karlin, Total Positivity, Stanford University Press, Stanford, Calif, USA, 1968.

[12] K. Jain, H. Singh, and I. Bagai, "Relations for reliability measures of weighted distributions," Communications in Statistics, Theory and Methods, vol. 18, no. 12, pp. 4393-4412, 1990.

[13] J. Bartoszewicz and M. Skolimowska, "Preservation of classes of life distributions and stochastic orders under weighting," Statistics \& Probability Letters, vol. 76, no. 6, pp. 587-596, 2006.

[14] N. Misra, N. Gupta, and I. D. Dhariyal, "Preservation of some aging properties and stochastic orders by weighted distributions," Communications in Statistics, Theory and Methods, vol. 37, no. 5, pp. 627-644, 2008.
[15] S. Izadkhah and M. Kayid, "Reliability analysis of the harmonic mean inactivity time order," IEEE Transactions on Reliability, vol. 62, no. 2, pp. 329-337, 2013.

[16] M. Kayid, I. A. Ahmad, S. Izadkhah, and A. M. Abouammoh, "Further results involving the mean time to failure order, and the decreasing mean time to failure class," IEEE Transactions on Reliability, vol. 62, no. 3, pp. 670-678, 2013.

[17] S. Izadkhah, A. H. Rezaei Roknabadi, and G. R. M. Borzadaran, "Aspects of the mean residual life order for weighted distributions," Statistics, vol. 48, no. 4, pp. 851-861, 2014.

[18] D. Yue and J. Cao, "Some results on the residual life at random time," Acta Mathematicae Applicatae Sinica, vol. 16, no. 4, pp. 435-443, 2000.

[19] X. Li and M. J. Zuo, "Stochastic comparison of residual life and inactivity time at a random time," Stochastic Models, vol. 20, no. 2, pp. 229-235, 2004.

[20] N. Misra, N. Gupta, and I. D. Dhariyal, "Stochastic properties of residual life and inactivity time at a random time," Stochastic Models, vol. 24, no. 1, pp. 89-102, 2008.

[21] S. M. Ross, Stochastic Processes, John Wiley, New York, NY, USA, 1996.

[22] R. E. Barlow and F. Proschan, Statistical Theory of Reliability and Life Testing, Silver Spring, Md, USA, 1981.

[23] X. Ling, P. Zhao, and P. Li, "A note on the stochastic properties of a scale change random effects model," Statistics \& Probability Letters, vol. 83, no. 10, pp. 2407-2414, 2013.

[24] E. Fagiuoli and F. Pellerey, "Mean residual life and increasing convex comparison of shock models," Statistics \& Probability Letters, vol. 20, no. 5, pp. 337-345, 1994.

[25] M. Shaked and T. Wong, "Preservation of stochastic orderings under random mapping by point processes," Probability in the Engineering and Informational Sciences, vol. 9, no. 4, pp. 563580, 1995.

[26] F. Belzunce, E. Ortega, and J. M. Ruiz, "The Laplace order and ordering of residual lives," Statistics \& Probability Letters, vol. 42, no. 2, pp. 145-156, 1999.

[27] M. Kayid and S. Izadkhah, "Mean inactivity time function, associated orderings and classes of life distributions," IEEE Transactions on Reliability, vol. 63, no. 2, pp. 593-602, 2014.

[28] C. Ma, "Convex orders for linear combinations of random variables," Journal of Statistical Planning and Inference, vol. 84, no. 1-2, pp. 11-25, 2000. 


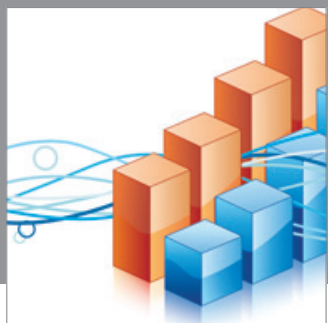

Advances in

Operations Research

mansans

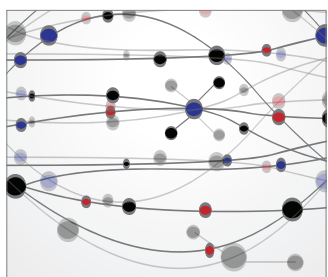

The Scientific World Journal
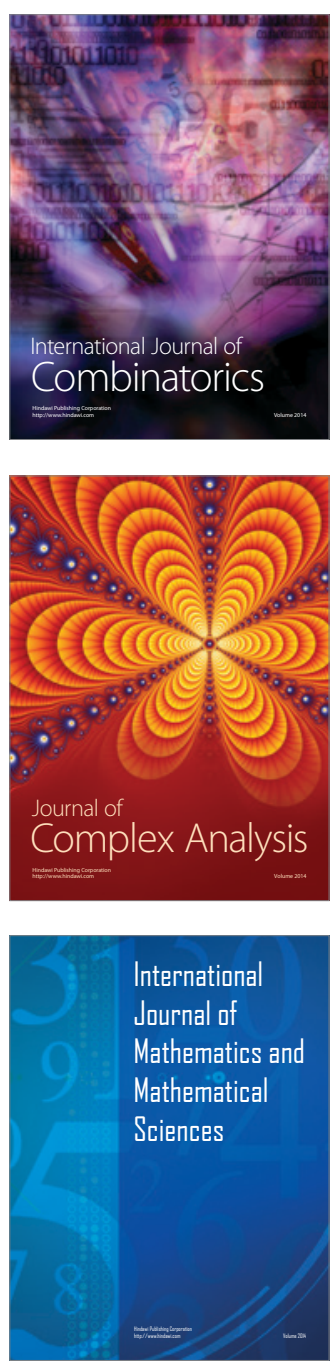
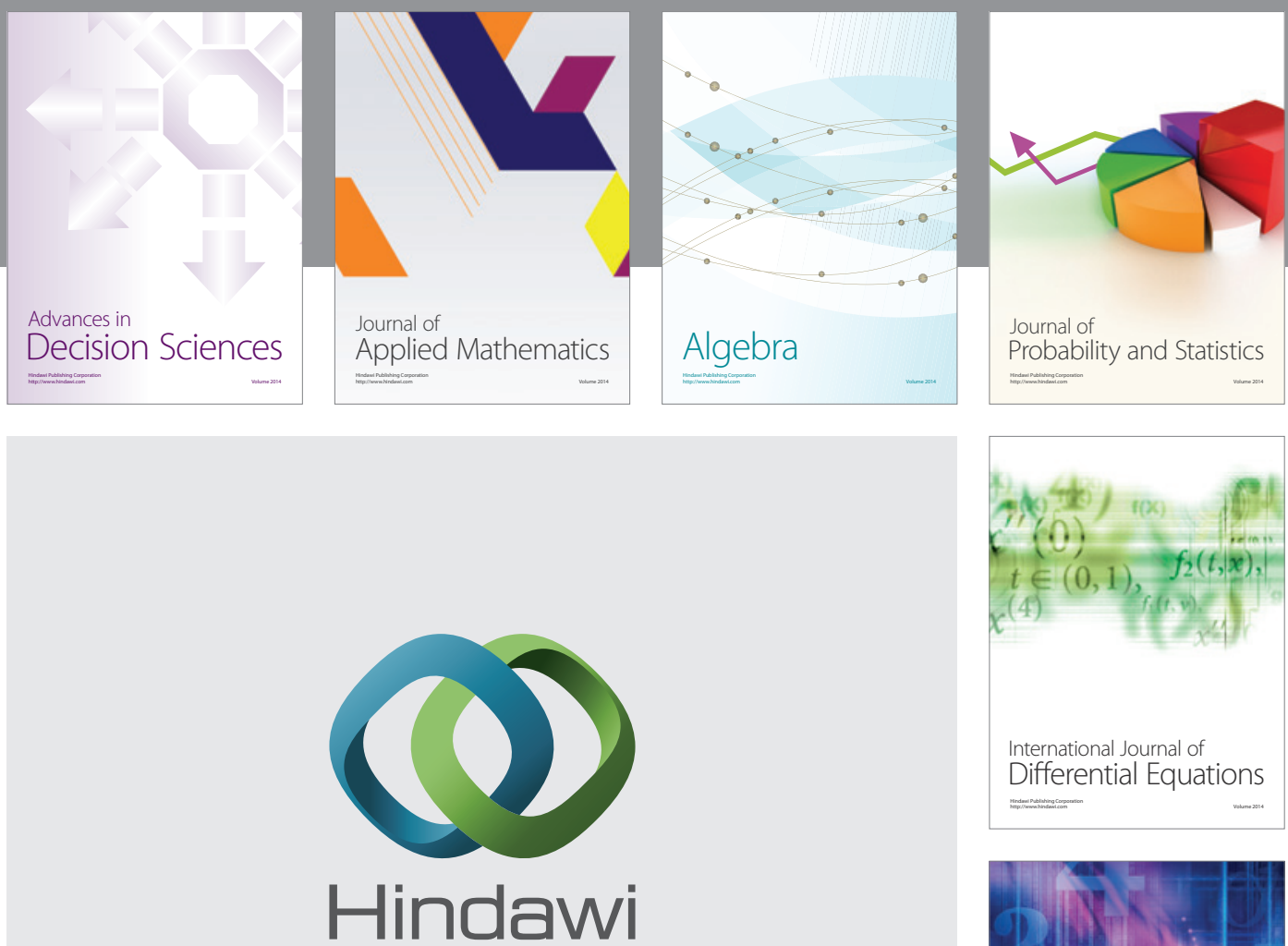

Submit your manuscripts at http://www.hindawi.com
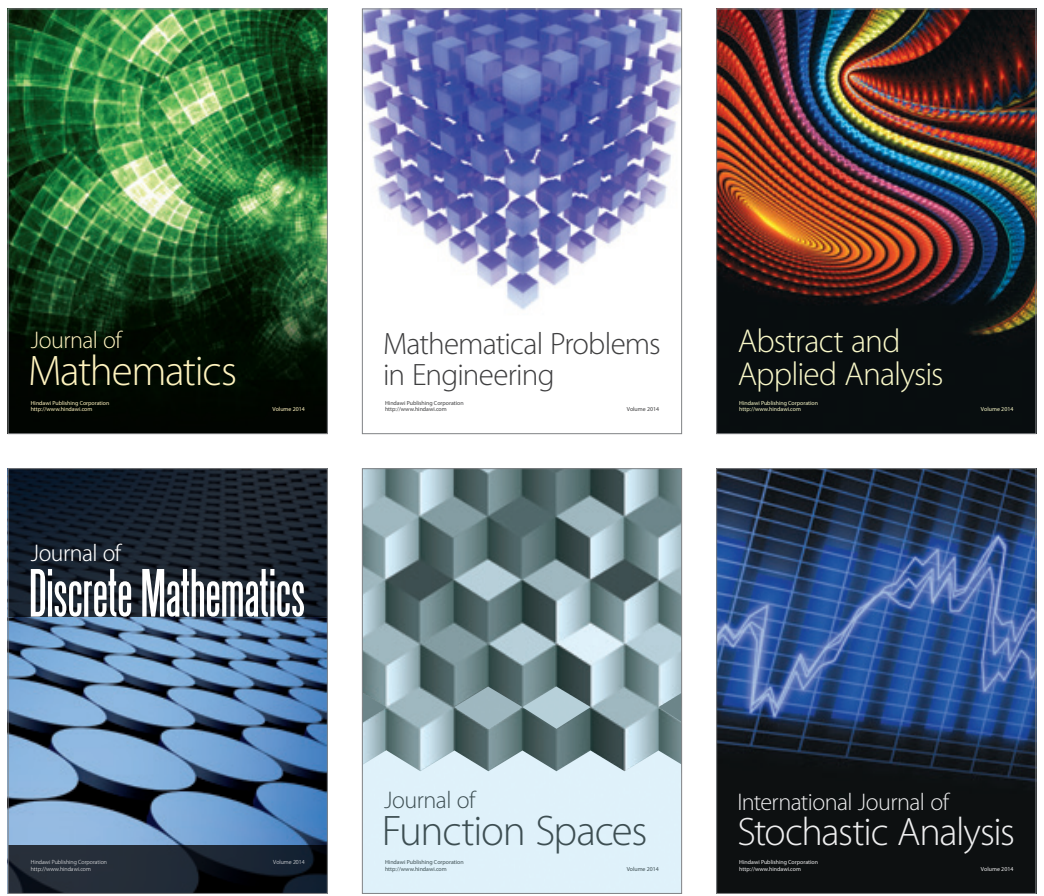

Journal of

Function Spaces

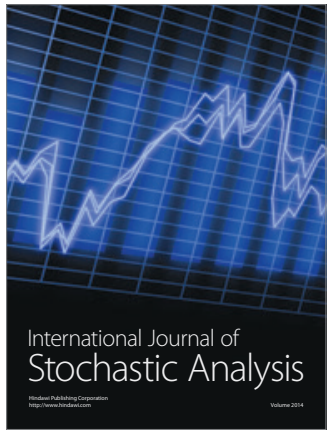

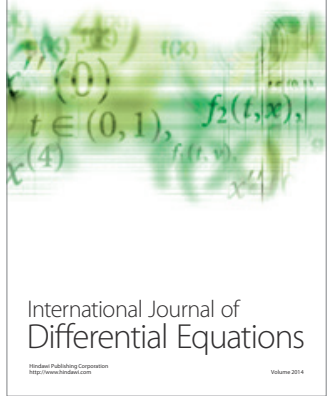
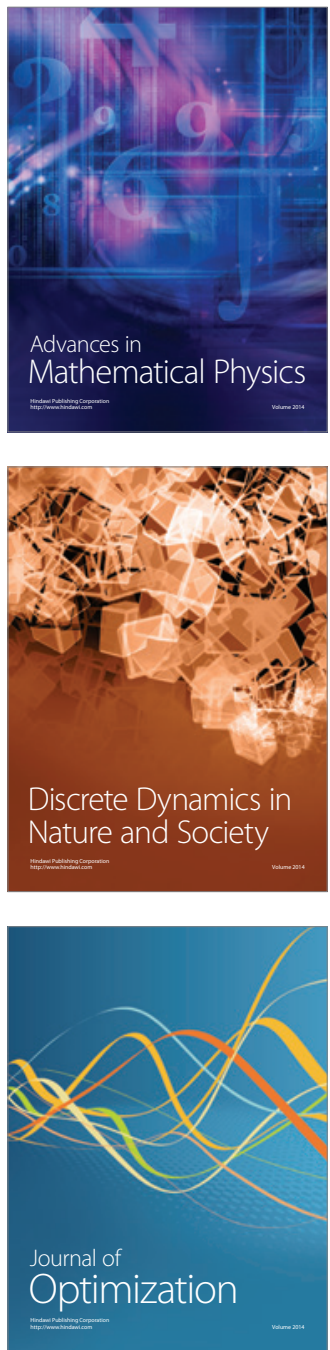